\title{
INTELLIGENT CONTROL OF BIPED ROBOT WITH HETEROGENEOUS LEGS
}

\author{
Binrui Wang*, Xinhe $\mathrm{Xu}^{*}$, Jindong Tan** \\ *Key Laboratory of Process Industry Automation, Ministry of Education, \\ Institute of AI and Robotics, Northeastern University, \\ Shenyang, 110004 China, E-mail: wangbinrui@tom.com \\ ** Department of Electrical and Computer Engineering, Michigan Technological \\ University, Houghton, MI 49931 USA, E-mail: jitan@mtu.edu
}

\begin{abstract}
Intelligent bionic leg (IBL) is important for above-knee amputees. Biped robot with heterogeneous legs (BRHL) including artificial leg and bionic leg is proposed as a good test-bed for IBL. Mechanism and virtual prototype of BRHL is designed and built. Model of BRHL is established. Based on the complex differential algebraic equation of bionic leg, computed torque and PD feedback control of joint angle are introduced for gait tracking. The iterative learning control is applied in conjunction with computed torque and PD control law for good tracking performance. Control simulation is done and shows intelligent control is good for BRHL. Copyright (C) 2005 IFAC
\end{abstract}

Keywords: Intelligent control, robot dynamics, modeling, computed torque control, learning control, hierarchical structures.

\section{INTRODUCTION ${ }^{1}$}

Intelligent bionic leg (IBL) controlled by micro processing unit (MPU) is an advanced intelligent prosthesis (IP). It can help above-knee amputee to walk like health human, and is a new application of automatic control theory in amputee rehabilitation (Datta and Howitt, 1998; Wang and Xu, 2004). Owing to MPU control, amputee with IP can change gait velocity optionally, run, walk on slope, ride bicycle, and gait is more like health human than common mechanical prosthesis. It is important and helpful to amputee and has good application value.

Human knee is composed of bone, ligament, muscle and nerve. Interface of thighbone and shinbone is erose, and there is scrolling and sliding between them (Siegmer Blumentritt and Hans Werner, 1997), that is illustrated in figure 1a. The instant centre of rotation (ICR) track is ' $\mathrm{J}$ ' curve. Joint of common biped robot is 2-bar link mechanism and centre of rotation is

\footnotetext{
${ }^{1}$ This study is supported by National Natural Science Foundation of China, ID60475036.
}

single illustrated in figure $1 \mathrm{~b}$. In order to simulate human knee function, 4-bar closed-chain knee mechanism is used in IBL as shown in figure 1c. The ICR of 4-bar knee is the point of intersection of ant-bar and post-bar prolongation, so is polycentric. 4-bar knee can get higher foot clearance with ground at smaller flexion angle and dynamic characteristic is more like human knee.

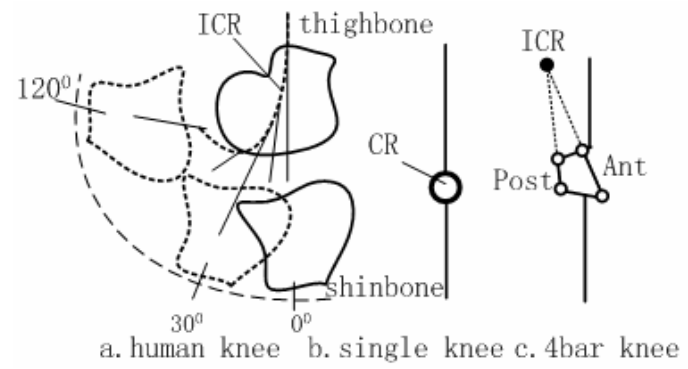

Fig. 1. The knees of human, common biped robot and 4-bar link.

Walking test of amputee fixed with intelligent bionic leg is important to IBL study. But in view of amputee physical limitation, plentiful and repeated walking test is difficult. At present, the leg simulator used in 
IP test is shown in figure 2.

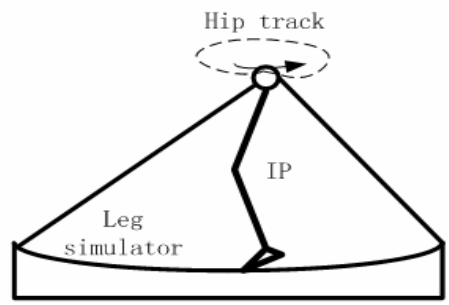

Fig. 2. Sample leg simulator.

This leg simulator can produce human hip motion and drive IP to test swing and stance performance (Kim, 2001). But it cannot be used to test the stability and harmony of amputee fixed with IP and the capability of IP following sound leg gait.

Biped robot can walk like human, so can be applied to test IBL. Based on this idea, a new-style biped robot with heterogeneous legs (BRHL) is proposed (Xu and Wang, 2004). An artificial leg (AL) of homogeneous biped robot, an intelligent bionic leg (BL) and simple head and torso (HAT) compose BRHL. Artificial leg including thigh, shin and foot, has 6 degrees of freedom (3 DOF hip, 1 DOF knee and 2 DOF ankle), and joints are driven by electromotor consuming larger energy. Bionic leg includes an artificial leg hip joint, intelligent bionic leg and flexible prosthetic foot. Prosthetic foot is fixed to IBL, so bionic leg has 4 degrees of freedom. HAT is smaller and simpler than human's. Legs mechanism dimension and joints motion space are designed according to $175 \mathrm{~cm}$ high male adult. BRHL virtual prototype, bionic leg work principle and BRHL model are presented in figure 3.

Bionic leg knee is semi-controlled by magneto-rheo-logical (MR) damper that is energy-saving. BRHL can be used to do plentiful and repeated test instead of amputee, so is a good test-bed for studying intelligent bionic leg. On the other hand, bionic leg can be used to study how to promote biped robot walking performance and make it more like human. Since IBL has self MPU, BRHL integrated by two intelligent agents can be used to study man-machine coordination.

After introduction, gait plan and coordination control method of BRHL are given. In the third part, dynamic model is established, and computed torque and PD control is studied for bionic control. In order to get good gait tracking, P-type closed-loop iterative learning control is given. In the fourth part, control simulation has been done and result is given. The conclusion is presented in the end.
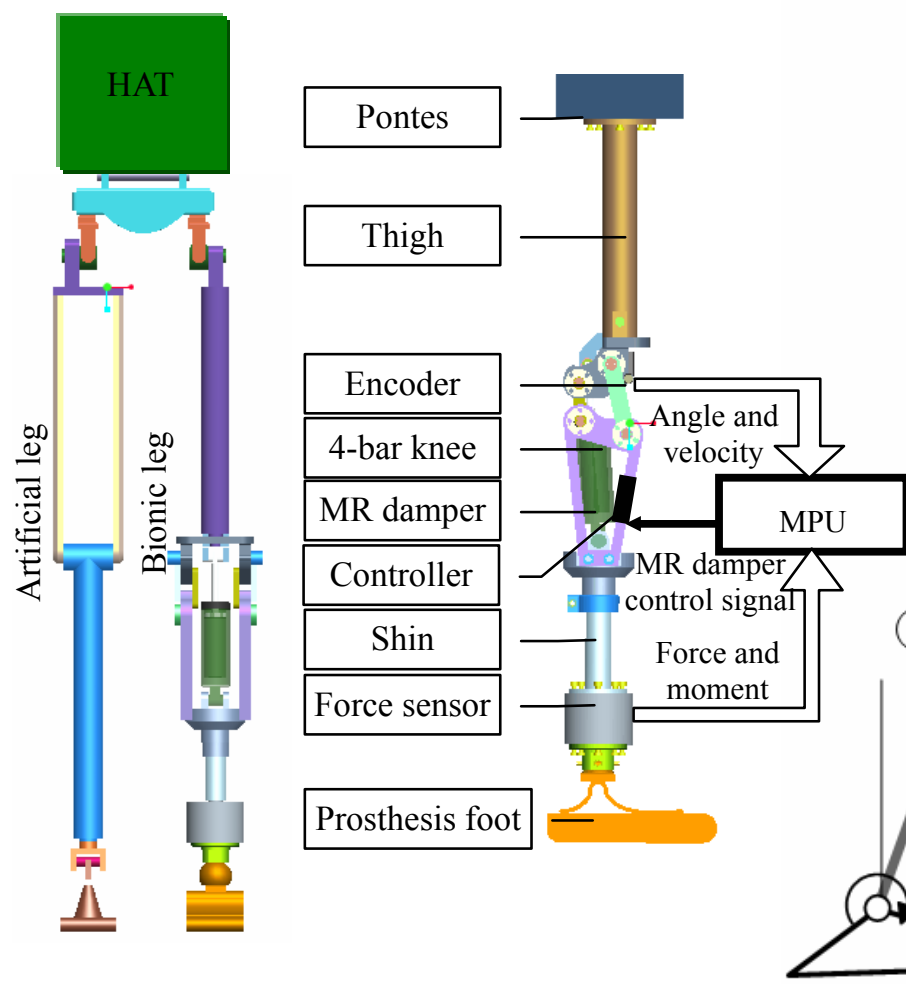

a. Virtual prototype.

b. Bionic leg work principle.

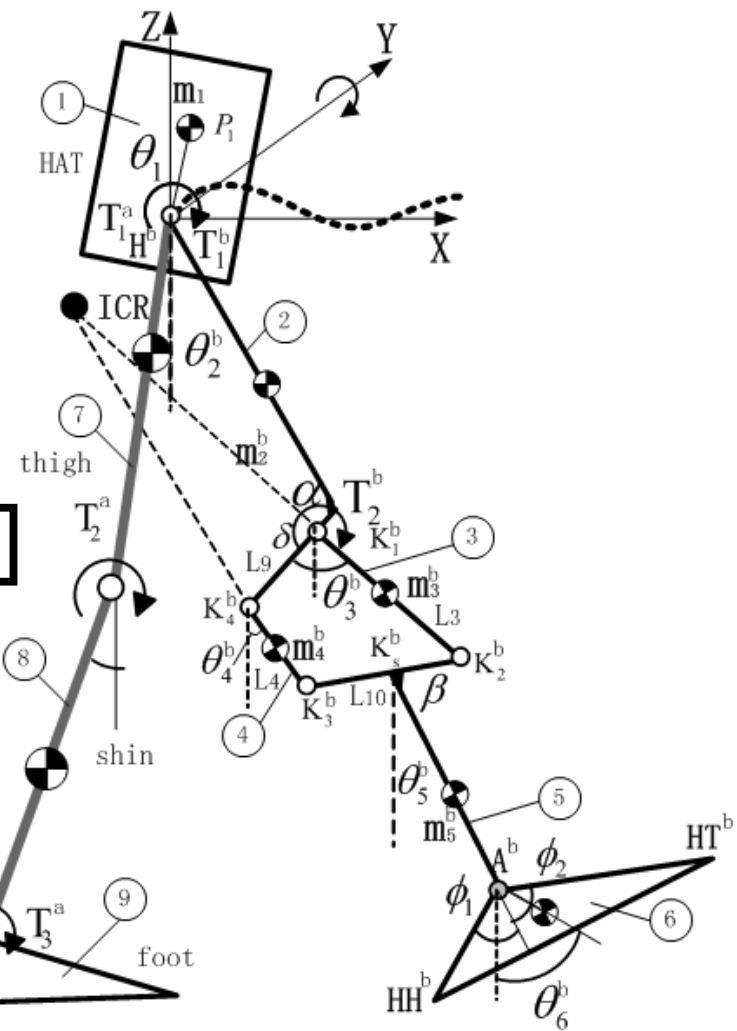

c. BRHL 9-link mode.

Fig. 3. BRHL virtual prototype, bionic leg work principle and BRHL model.

\section{BRHL INTELLIGENT CONTROL STRUCTURE}

BRHL is a complex, nonlinear and strong coupling system and is naturally unstable. Three layers hierarchical architecture control is good for BRHL control that is depicted in table 1. 
In BRHL bionic leg is an intelligent agent; the rest is another intelligent agent called artificial leg. Artificial leg agent can be regarded as the simulator of above-knee amputee.

At first, study is focused on the steadily walking of BRHL. Dual-leg's coordination is the key problem. The master/slave coordination method is applied in
BRHL dual-leg's coordination. Artificial leg is defined as master leg, bionic leg as slave leg. It is the same with work principle of amputee's sound leg and IP. Slave leg tracks master leg subject to kinematics and dynamics constrains. So bionic leg agent has not task layer and its desired gait is artificial leg's gait.

\section{$\underline{\text { Table 1. Depiction of three layers hierarchical architecture control }}$}

\begin{tabular}{llll} 
& Task layer & Plan layer & Drive layer \\
\hline Intelligent grade & High & Middle & Low \\
Task & $\begin{array}{l}\text { According to environment } \\
\text { information or human command, } \\
\text { decide what to do. }\end{array}$ & $\begin{array}{l}\text { Plan the gait for finishing } \\
\text { the desired task. }\end{array}$ & $\begin{array}{l}\text { Drive joint to produce } \\
\text { planed gait. }\end{array}$ \\
$\begin{array}{l}\text { Artificial leg } \\
\text { agent equipment }\end{array}$ & $\begin{array}{l}\text { ADVANTECH industrial } \\
\text { computer 610 }\end{array}$ & $\begin{array}{l}\text { ADVANTECH industrial } \\
\text { computer 610 }\end{array}$ & $\begin{array}{l}\text { Googol motion control } \\
\text { board and PMAC driver }\end{array}$ \\
$\begin{array}{l}\text { Bionic leg agent } \\
\text { equipment }\end{array}$ & TI DSP TMS320 F240 & $\begin{array}{l}\text { RD1005 MR damper } \\
\text { wonderful box }\end{array}$ \\
\hline
\end{tabular}

Human walking gait can be measured and recorded using human motion detection system. Because BRHL leg's design is according to human leg, human gait can be directly used and satisfy the condition of zero moment point (ZMP) in steady stance area (Anirvan and Yashihiko, 1999). Classified gait data, joints angle, on different walking velocity is stored in gait database, and is used as BRHL desired gait. The knee relative angle of typical human gait is illustrated in figure 4. Whole gait is divided into stance phase and swing phase.

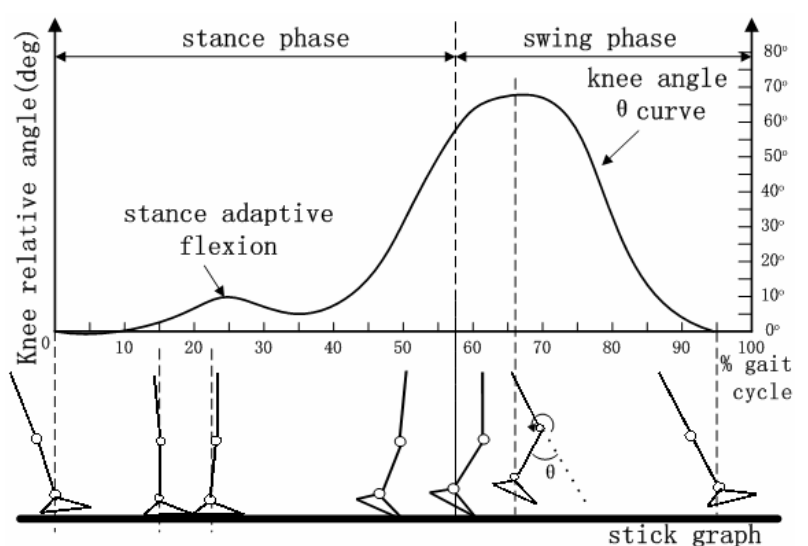

Fig. 4. Typical gait of human knee.

\section{INTELLIGENT CONTROL OF BRHL}

\subsection{BRHL Modeling}

Dynamic model is the foundation of system analysis and control. BRHL is modeled as 9 link system which plane model is illustrated in figure 3c. Artificial leg is 3 links model and is widely study. Bionic leg is 4-bar close-link parallel model, and is more complex than artificial leg.

Variables with superscript ' $a$ ' denotes artificial leg, and variables with superscript ' $\mathrm{b}$ ' bionic leg. $\theta_{i}$ are generalized coordinate. $l_{i}$ are link bar length. Only important variables are marked in figure $3 \mathrm{c}$. Bionic knee 4-bar close-link constrain is written as

$$
f:-l_{9}^{b} e^{j\left(\theta_{2}^{b}-\alpha\right)}-l_{3}^{b} e^{j \theta_{3}^{b}}+l_{10}^{b} e^{j\left(\theta_{5}^{b}-\beta\right)}+l_{4}^{b} e^{j \theta_{4}^{b}}=0
$$

BRHL can be divided into artificial leg subsystem $\Sigma_{a}$ and bionic leg subsystem $\Sigma_{b}$. From crotch center, two leg's models are established apart. The motion of crotch center is represented as

$$
M_{\text {crotch }}=\frac{M_{h}^{a}+M_{h}^{b}}{2}
$$

where $M_{h}^{a}$ and $M_{h}^{b}$ are motion of hip center, respectively. Since crotch rotation is small, $M_{\text {crotch }}$ is simplified as

$$
M_{h}^{a}=M_{h}^{b}=M_{\text {crotch }}
$$

Using Lagrangian method (Yin, et al., 2001), establish each leg model as equation (3) and (4). Where $F\left(M_{\text {crotch }}\right)$ is the item being relational with $M_{\text {crotch }}$. $M(\theta)$ is the mass and mass moment of inertia matrix; $C(\theta, \dot{\theta})$ is the vector of generalized centrifugal and Coriolis forces; $G(\theta)$ is the vector of the gravitational forces; $B$ is the coefficient matrix 
of applied torques. $\dot{f}_{\theta^{b}} \lambda$ is the constrain torque.

$$
\dot{f}_{\theta^{b}}=\frac{\partial f}{\partial \theta^{b}}, T^{a}=\left[\begin{array}{lll}
T_{1}^{a} & T_{2}^{a} & T_{3}^{a}
\end{array}\right]^{T}, T^{b}=\left[\begin{array}{ll}
T_{1}^{b} & T_{2}^{b}
\end{array}\right]^{T}
$$

Obviously using apart modeling, each leg's model is established with self variables. $F\left(M_{\text {crotch }}\right)$ is the coupling item of two legs and is easily gained and separated.
In order to stably walking, slave leg must track master leg gait in a reasonable phase-different. Gait phase-different is written in equation (5). $\left[T_{\min }, T_{\max }\right]$ is allowable phase-different scope. The simultaneous equation of equation (3), (4) and (5) is the BRHL coordination model.

$$
\begin{aligned}
& \sum_{a}: M^{a}\left(\theta^{a}\right) \ddot{\theta}^{a}+C\left(\theta^{a}, \dot{\theta}^{a}\right)+G\left(\theta^{a}\right)+F^{a}\left(M_{\text {crotch }}\right)=B^{a} T^{a} \\
& \sum_{b}: M^{b}\left(\theta^{b}\right) \ddot{\theta}^{b}+C\left(\theta^{b}, \dot{\theta}^{b}\right)+G\left(\theta^{b}\right)+F^{b}\left(M_{\text {crotch }}\right)=B^{b} T^{b}+\dot{f}_{\theta^{b}} \lambda \\
& \theta^{b}(t)=\theta^{a}(t-\Delta t) \quad T_{\min } \leq \Delta t \leq T_{\max }
\end{aligned}
$$

Because of two legs' mechanism different, their dynamic models and control methods are not the same. Firstly, on the assumption that crotch point motion is ideal and symbolized as $M_{c r o t c h}^{*}$, two legs' controls are studied respectively. Artificial leg control can use biped robot control method. Bionic leg model is more complex and more difficult to solve with constrain torque item $\dot{f}_{\theta^{b}} \lambda$, so its control is complicated than artificial leg and is the key problem of BRHL study. Following study is focused on bionic leg control.

\subsection{The computed torque and PD control}

Due to constrain torque $\dot{f}_{\theta^{b}} \lambda$, dynamic model of bionic leg sub-system $\Sigma_{a}$ is differential algebraic equation and is more difficult to solve. Considering 4-bar knee mechanism size is much smaller than thigh and calf length, bionic leg model can be simplified with omitting $\dot{f}_{\theta^{b}} \lambda$. Based on this simplified model, computed torque and PD feedback control method are introduced to track the desired gait. The nonlinear terms in the dynamic model is eliminated by the computed torque terms.
Based on simplified model, define

$$
T^{b}=\alpha T^{\prime}+\beta
$$

where $\alpha=\left(B^{b}\right)^{-1} M^{b}\left(\theta^{b}\right)$, and

$$
\beta=\left(B^{b}\right)^{-1}\left(C\left(\theta^{b}, \dot{\theta}^{b}\right)+G\left(\theta^{b}\right)+F^{b}\left(M_{\text {croth }}^{*}\right)\right)
$$

$T^{\prime}$ is written as

$$
T^{\prime}=\ddot{\theta}^{b}=I \ddot{\theta}^{b}
$$

This is a non-coupling system, and the relation between input $T^{\prime}$ and output $\ddot{\theta}^{b}$ is linear. So PD feedback control can be used. Control signal is written as

$$
u=\ddot{\theta}_{d}^{b}+K_{v} \dot{E}+K_{p} E=T^{\prime}
$$

where $\ddot{\theta}_{d}^{b}$ is the desired joint angle acceleration of bionic leg.

$$
E=\theta_{d}^{b}-\theta^{b}
$$

On the condition of $\theta^{b}(0)$ and $\dot{\theta}^{b}(0)$ being in accord with the desired initial setting, the desired gait can be realized by this control method, in theory. Computed torque and PD control is shown in figure 5.

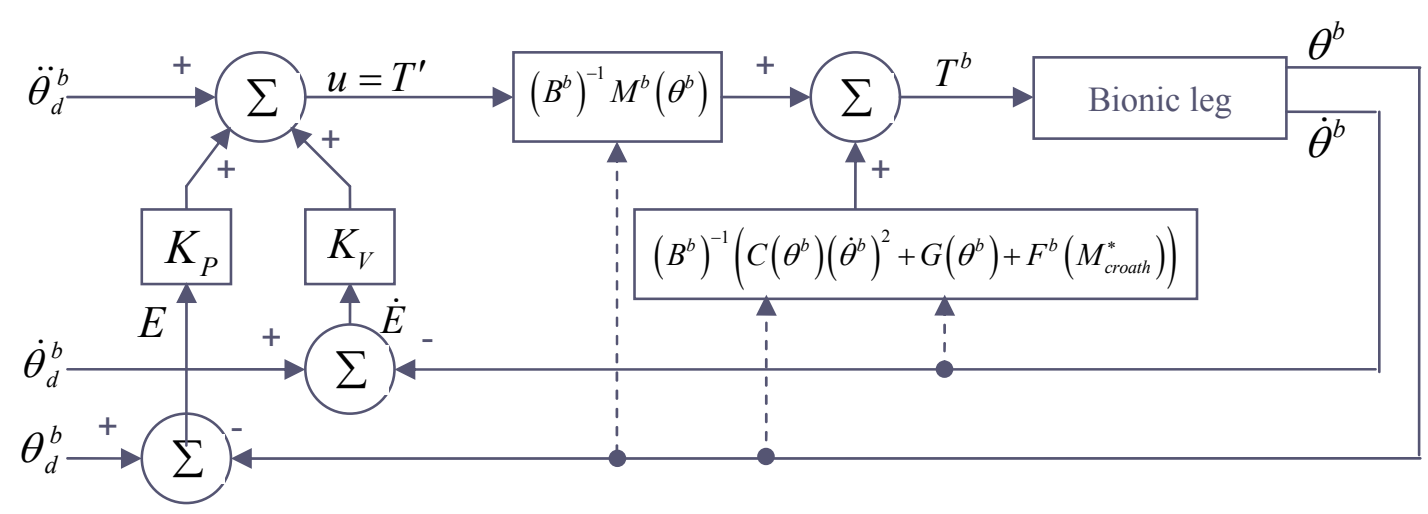

Fig. 5. Computed torque and PD feedback control. 
The control input of bionic leg is written as

$$
T^{b}=\left(B^{b}\right)^{-1} M^{b}\left(\theta^{b}\right)\left(\ddot{\theta}_{d}^{b}+K_{v} \dot{E}+K_{p} E\right)+\left(B^{b}\right)^{-1}\left(C\left(\theta^{b}, \dot{\theta}^{b}\right)+G\left(\theta^{b}\right)+F^{b}\left(M_{\text {croth }}^{*}\right)\right)
$$

On account of modeling error and constrain torque ignored in model, the model control method can not ideally track joints angle.

\subsection{The iterative learning control}

Bionic (slave) leg tracks artificial (master) leg's gait is a dynamic tracking problem. Because of gait's periodic repetition, iterative learning control (ILC) is a good control method to bionic leg. The P-type close-loop iterative learning control applied in bionic leg control is represented as

$$
u_{k+1}(t)=T_{k+1}^{b}(t)=u_{k}(t)+\Gamma_{p}(t) e_{k+1}(t)
$$

where $\Gamma_{p}$ is a gain of learning

$$
e_{k}(t)=\theta_{d}^{b}(t)-\theta^{b}(t)
$$

In order to get good tracking performance, the initial constrain must be satisfied, that is written as

$$
u_{k}(0)=u_{d}(0)
$$

In terminal swing phase and initial stance phase of gait cycle, bionic leg knee is full extended, as shown in figure 4. If initial state of ILC is selected in those phase, initial constrain is easily meet.

According to reference (Ling and Wang, 1995; Richard, 2000; Wu, et al., 2001), the convergence condition of ILC is written as

$$
\rho\left[\left(1+\Gamma_{p}(t)\right)^{-1}\right]<1
$$

ILC is not based on model, so it resists the modeling error. For gaining good tracking performance of bionic leg, a new control scheme is proposed which integrates computed torque and PD feedback control with iterative learning control, and sets computed torque and PD control signal as $u_{0}(k)$ of ILC.

\section{CONTROL SIMULATION}

The bionic leg and test frame built for BRHL study are presented in figure 6 .

In order to testing control method, BRHL virtual prototype is established using software Adams (Mechanical Dynamics INC), as shown in figure 1a. Control module is built in MATLAB Simulink. Test gait data is obtained from software APAS (Ariel Dynamics Inc), and joints angle curves are presented in figure 7. Sampling interval is $0.02 \mathrm{~s}$

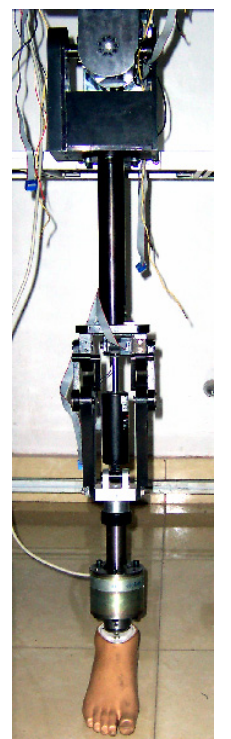

Fig. 6. Bionic leg.
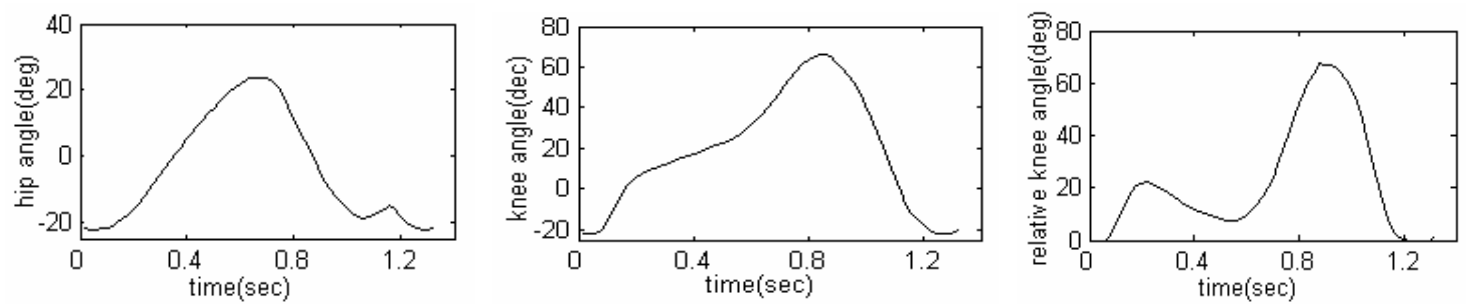

Fig. 7. Typical human gait used as desired joint angle.

Conjunction control of computed torque and PD feedback control with iterative learning control of bionic knee is simulated in swing phase and initial stance phase. Simulation result is illustrated in figure 8. The initial tracking curve is from the result of computed torque and PD feedback control.

Simulation results show that control can get good tracking performance of bionic leg 4-bar knee. Large tracking error was found at point $\mathrm{A}$, when foot 
contacting ground. It is because the impact between foot and ground.

The perfect control methods for two legs respective are the foundation of dual-leg's coordination. The different between respective control and dual-leg's control is that the coupling terms must be considered. This is the reason of separating $F\left(M_{\text {crotch }}\right)$ from coordination model.

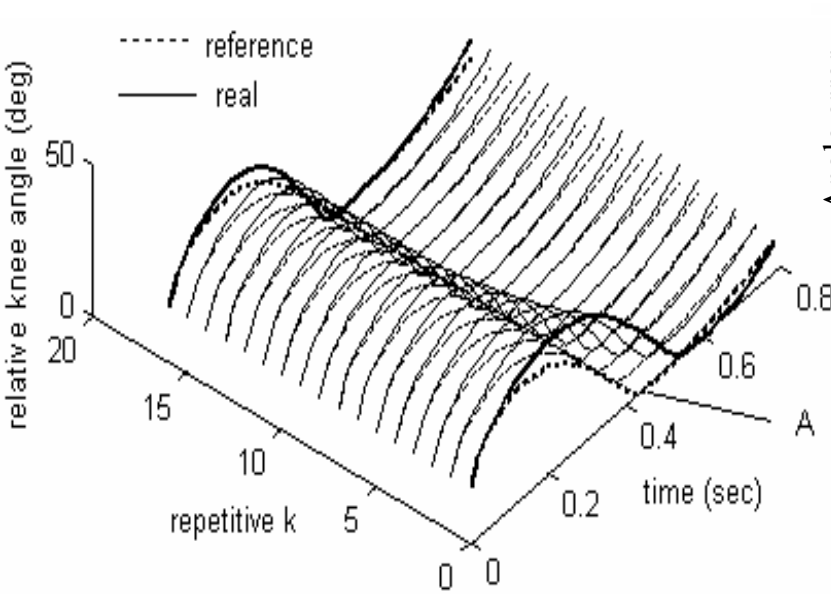

a. relative knee joint angle tracking
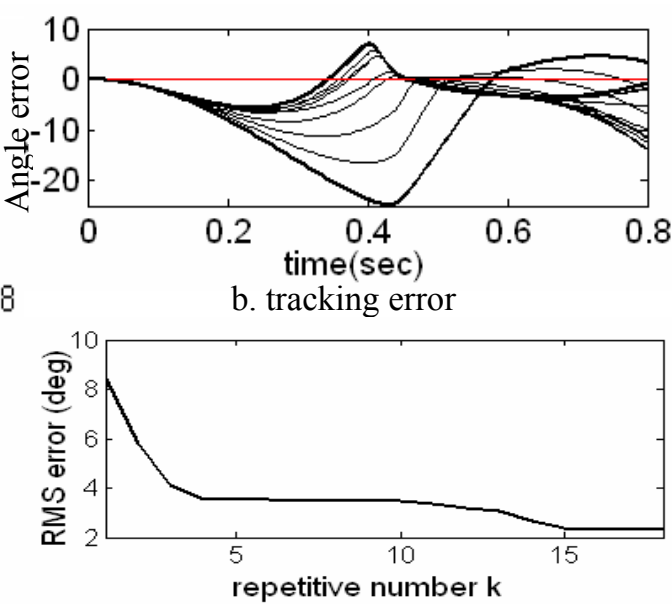

c. RMS tracking error

Fig. 8. The simulation results of $\mathrm{P}$ close-loop iterative learning control.

\section{CONCLUSION}

In this study, a new-type robot BRHL is proposed, that is designed to simulate amputee walking with intelligent bionic leg and is a good test-bed for IBL. With polycentric 4-bar knee, biped robot is more humanoid. BRHL virtual prototype is designed. Aim at bionic leg nonlinear model with constrains, computed torque and PD control and P-type closed-loop iterative learning control are mainly studied. Above work give a good foundation for BRHL study. As a future work, dual-leg's synchronous walking control should be carried out and is more difficult when artificial leg can not ideally imitate the human walking. A control method for resisting the impact of ground reaction force should be studied. Since the bionic leg is different with artificial leg in mechanism, driver and energy supply, it cannot follow all gait of artificial leg. So the optimal control should be studied subject to the best gait tracking performance.

\section{REFERENCES}

Anirvan and Yashihiko (1999). Making feasible walking motion of humanoid robots from human motion capture data. Detrott: Proceedings of the 1999 IEEE International Conference on Robotics \& Automation, pp.1044-1049.

Datta, D. and J. Howitt(1998). Conventional versus microchip controlled pneumatic swing phase control for trans-femoral amputees: User's verdict. Prosthetics and Orthotics International, 22, pp.
129-135.

Kim J.H. (2001). Development of an Above knee prosthesis using MR damper. Proceeding of the 2001 IEEE international conference on robotics \& automation, Seoul, pp.3686-3691.

Ling H. and Wang L. (1995). Convergence of P type close-loop iterative learning control for nonlinear system. Control Theory and Applications, 12, pp. 742-745.

Richard W. (2000). Iterative learning control and repetitive control for engineering practice. INT. $J$. CONTROL, 73, pp. 930-954.

Siegmer Blumentritt and Hans Werner (1997). Design Principles, Biomechanical Data and Clinical Experience with a Polycentric Knee Offering Controlled Stance Phase Knee Flexion: A Preliminary Report. Journal of prosthetics and orthotics, 9, pp. 18-24.

Wang B.R. and Xu X.H. (2004). Study of Intelligent Bionic Limb Prosthesis. Control \& Decision, 19, pp.121-127.

Wu H.Y., Zhou Z.Y. and Xiong S.S. (2001). D-type iterative learning control with application to FNS limb motion control system. Control Theory and Applications, 18, pp.409-413.

Xu X.H., Wang B.R. and Tan J.D. (2004). A New Kind of Robot Model ----- Biped Robot with Heterogeneous Legs. High Technology Letters, 14, pp.55-58.

Yin, Y.K., H. Cheng, Z.H. Xiong, G.F. Liu and Z.X. $\mathrm{Li}$ (2001). On the dynamics of parallel manipulators. Proceeding of the 2001 IEEE, Seoul, pp. 3766-3771. 